\title{
Analysis of MHD Forced Convective Flow of Variable Fluid Properties over a Saturated Porous Medium with Thermal Radiation Effect
}

\author{
Kolawole Sunday Adegbie ${ }^{1, \mathrm{a}}$, Adeyemi Isaiah Fagbade ${ }^{2, b *}$ \\ ${ }_{1,2}$ Department of Mathematical Sciences,School of Sciences Federal University of \\ Technology,P.M.B. 704,Akure Ondo State, Nigeria \\ ${ }^{1}$ ksadegbie@futa.edu.ng, ${ }^{2 *}$ aifagbade@futa.edu.ng
}

\begin{abstract}
Keywords: Forced convection, Magnetohydrodynamic, Variable fluid viscosity, thermal conductivity,
\end{abstract} Bivariate spectral Relaxation method, Thermal radiation, saturated porous medium.

\begin{abstract}
The present paper addresses the problem of MHD forced convective flow in a fluid saturated porous medium with Brinkman-Forchheimer model, which is an important physical phenomena in engineering applications. The paper extends the previous models to account for effects of variable fluid properties on the forced convective flow through a porous medium in the presence of radiative heat loss using bivariate spectral relaxation method (BSRM). The dynamic viscosity $\mu$ and thermal conductivity of the newtonian fluid are assumed to vary linearly respectively, with temperature whereas the contribution of thermal radiative heat loss is based on Rosseland diffussion approximation. The flow model is described and expressed in form of a highly coupled nonlinear system of partial differential equations. The method of solution BSRM as proposed by Motsa [25] seeks to decouple the original system of PDEs to form a sequence of equations that can be solved in a computationally efficient manner. BSRM is an approach that applies spectral collocation independently in all underlying independent variable is executed to obtain approximate solutions of the problem. The proposed algorithm is supposed to be a very accurate, convergent and very effective in generating numerical results. The results obtained show a significant effects of the flow control parameters on the fluid velocity and temperature respectively. Consequently, the wall shear stress and local heat transfer rate of the present paper are compared with the available results in literatures. Remarkable impacts and a good agreement are found.
\end{abstract}

\section{Introduction}

Theoretical and applied research in fluid flow, heat and mass transfer in porous media has received remarkable attention over the past three decades. This is due to the importance and relevance of this research area in many engineering applications. Significant advances have been made in modeling fluid flow, heat and mass transfer through a porous medium including clarification of many important physical phenomena. For instance, the non-darcy effects on momentum, energy amd mass transport in porous media have been studied in depth for various geometrical configurations and boundary conditions.Many of the research works in porous media for the past couple of decades uses what is now known as the Brinkman-Forchheimer-extended darcy or simply refer as generalized model by Vafai [1].

Magnetohydrodynamic forced convective flows in a fluid saturated porous media are of great value in various engineering, scientific and industrial applications in heat and mass transfer which occurs in the fields of design of chemical processing equipment,formation and dispersion of fog,distributions of temperature and moisture over agricultural fields and groves of fruit trees and damage of crops due to freezing and pollution of the environment, grain storage systems, heat pipes, packed microsphere insulation, distillation towers, ion exchange column, subterranean chemical waste migration system,solar power absorbers etc. A sizeable number of studies have been reported in literature with focus and emphasis on the problem of combined heat and mass transfer in porous media. The qualitative analysis of convective transport in a porous medium in the presence of non-darcian effects has also been a subject 
of investigation in the recent years. Due to its important application in many field, a full understanding for combined heat and mass transfer by non-darcy natural convection from a heated flat surface embedded in fluid saturated porous medium is very important and meaningful. The inertia effect is significant in any flow field at a higher flow rate and it can be accounted for through the addition of a velocity squared term in the momentum equation. This is known as the Forchheimer's extension of darcy law. A detailed review of convective heat transfer in darcian and non-darcian porous media including an exhaustive list of references can be found in the book by Nield and Bejan [2] and Srinivasacharya et. al [6], Ingham and Pop [3] and Pop and Ingham [4] reviewed a good number of articles in their books.

These revealed the level of understanding on the momentum, heat and mass transfer phenomena in porous media. However, many of the previous literatures in this area of research work have been on the investigation of either convection in plane walls or in a channel bounded by the porous medium. Till date there are very limited research works on convective heat and mass transfer from heated bodies of higher complexity, such as circular cylinder embedded in saturated porous medium. Magnetohydrodynamic flow, heat and mass transfer from a horizontal cylinder immersed in a fluid saturated porous medium have practical importance in engineering applications such as seen in compact heat exchangers, solar power collectors and nuclear reactors. El-Amin [5] investigated a combined effect of viscous dissipation and joule heating on MHD forced convection flow over a non-isothermal horizontal cylinder embedded in a fluid saturated porous medium. The influence of both first and second order resistance due to the solid matrix of non-darcy porous medium,joule heating and viscous dissipation on forced convection flow over a horizontal cylinder under the action of transverse magnetic field were adequately examined with constant dynamic viscosty and thermal conductivity in the absence of thermal radiation.

Cheng [7] studied the combined heat and mass transfer in natural convection flow from a vertical wavy surface in a power-law fluid over a saturated porous medium with thermal and mass stratification. Mukhopadhyay [8] examined the effects of thermal radiation on unsteady mixed convection flow and heat transfer over a porous stretching surface in porous medium.

Pantokratoras [9] made a startling theoretical study on the effect of variable viscosity on the flow and heat transfer over a continuous plate. Adegbie and Alao [10] obtained an exact analytical solutions for the solution of temperature-dependent viscous fluid between parallel heated walls in the presence of viscous dissipation. Ahmad et al. [11] investigated non-similar solutions of mixed convection boundary layer flow past an isothermal horizontal circular cylinder with temperature-dependent viscosity. they concluded that flow and heat transfer characteristics are significantly influenced by temperature-dependent viscosity. It is remarkable to note that the study of magnetohydrodynamic flow has an important application in engineering and industries and may also be used to deal with the problem such as cooling of nuclear reactors by liquid sodium and induction flow meter, which is determine by the potential difference in the fluid in the direction perpendicular to the motion of fluid and the magnetic field, see Ganesan [12].

Thermal radiation is one of the fundamental mechanisms of heat transfer and its influence plays a significant role in controlling heat transfer process in polymer processing industry. The quality of final product depends to a large extent on the amount of heat energy such object is exposed to and the level of heat controlling factors. Thermal radiation occurring within these systems is as a result of thermal emission by the hot walls and working fluid. These effects become more significant especially when the temperature difference between the wall surface and the ambient temperature is large. At high operating temperature, thermal radiation effects can be quite significant and the knowledge of radiation heat transfer is very important for the design of pertinent equipment Seddeek [13]. Hence thermal radiation is one of the vital factors controlling the heat and mass transfer Dulal [14]. Observing various researches into the dynamics of MHD flow, it is seen that there are varying focuses on terms like viscosity and thermal diffusivity, heat generation or absorption coefficient, magnetic induction, electrical conductivity, Eckert number, heat dissipative term and a host of others. According 
to Mahmoud [15], in most studies, the viscosity and thermal conductivity of the working fluid are usually assumed a constant value. In recent development, Promise [16] examined steady-state solution to Magnetohydrodynamic thermally radiating and reacting thermo-solutal viscous flow through a channel with porous medium. The chemical reaction is assumed to be strongly exothermic under the generalized Arrhenius kinetics neglecting the consumption of the material. An approximate solutions were obtained for the model's governing equations using WKBJ approximations. The results obtained were observed to be relative on the dimensionless controlling parameters.

Salema and Fathy [19] examined the effects of variable properties on MHD heat and mass transfer flow near a stagnation point towards a stretching sheet in a porous medium with thermal radiation. Minkowyez and Haji-Sheikh [20] analyzed heat transfer in parallel plate and circular porous passage with axial conduction. The study of the problem of mixed convection flow from a vertical flat plate with temperature-dependent viscosity was examined by Hossain [21]. Non-darcy unsteady mixed convection flow near the stagnation point on a heated vertical surface embedded in a porous medium with thermal radiation and variable viscosity was investigated by Hassanien [22]. Recently, Sharma [23] delved into the problem of MHD flow, heat and mass transfer about a horizontal cylinder in a saturated porous medium. The previous investigations on the field of MHD flow provide some clarifications and insights in understanding several pertinent aspects of modeling of transport phenomena in porous media but without combined effects of variable viscosity and thermal conductivity in the presence of thermal radiation. In most of the previous investigations of these types of problem, the viscosity and thermal conductivity of the working fluid were assumed to be of constant value without consideration for heat loss by thermal radiation (see references cited).

However,it is an established fact that these thermo-physical properties change significantly with temperature variation. When variable viscosity and thermal conductivity of the fluid are taken into account in the transport phenomena through a porous medium, the flow characteristics are significantly changed as compared to a constant property case of the fluid. Thus to accurately predict the flow behaviour, it is necessary to take into account the variation of viscosity and thermal conductivity of fluid with temperature. Consequently, the present study extends the work of El-Amin [5] to examine the problem of MHD forced convection flow over a non-isothermal horizontal cylinder embedded in a fluid saturated porous medium with variable viscosity $\mu(T)$ and thermal conductivity $\lambda(\mathrm{T})$, which are modeled as a function of temperature, in the presence of thermal radiation. The governing boundary layer equations are transformed using suitable similarity variables to yield a highly coupled non-linear system of partial differential equations. The transformed governing equations are approach using spectral relaxation method. Spectral relaxation method is a newly proposed numerical scheme often use to solve non-linear systems of boundary value problems.

The method is developed by defining a rule of solution expression based on bivariate Lagrange interpolation. The spectral relaxation method algorithm is applied to decompose the governing nonlinear PDEs into a sequence of linear PDEs. The resulting linear sequence of PDEs contains variable coefficients and is impossible to solve exactly. Consequently, the Chebyshev spectral collocation method with bivariate Lagrange interpolation is applied independently in the space and time independent variables Motsa et al. [27]. In view of the application of the combination of bivariate interpolation and spectral collocation differentiation, Motsa et al. [25] called the new method bivariate spectral relaxation method (BSRM).

\section{Governing Equations}

Consider a steady two-dimensional magnetohydrodynamic flow of a viscous incompressible electrical conducting forced convection flow from a horizontal impermeable circiular cylinder with radius $r$ saturated in a porous medium with variable dynamic viscosity and thermal conductivity in the presence of thermal radiation. In order to account for the variation in thermo-physical properties of the fluid, it is valid to consider the mathematical model of temperature dependent viscosity model used in Sivagnana 
et al. [28] which was developed using the experimental data reported in (Batchelor (1987)) together with the mathematical model of temperature dependent thermal conductivity model of Charraudeau (1975) as

$$
\begin{aligned}
& \mu(T)=\mu_{\infty}\left(1+\gamma\left(T_{w}-T\right)\right), \\
& \lambda(T)=\lambda_{\infty}\left(1+\alpha\left(T-T_{\infty}\right)\right) .
\end{aligned}
$$

Here $\alpha$ and $\gamma$, are constants and their values depend on the reference states $\mu_{\infty}$ and $\lambda_{\infty}$, and thermal properties of the fluid. In general, It is worth noting that if $\gamma>0$ the flow model is designed for liquids and if $\gamma<0$ the flow model is designed for gases. $T_{w}$ and $T_{\infty}$ (with $T_{w}>T_{\infty}$ ) are the surface temperature and free stream temperature respectively. The operation of the flow in saturated porous medium requires high Reynold number and small Prandtl number respectively. The surface temperature of the working fluid is assumed to vary with the stream-wise direction of the flow, i.e.

$$
T_{w}-T_{\infty}=d(1-\cos \omega)^{p}, \quad 0 \leq \omega \leq \pi,
$$

where $d$ and $p$ are constants. We notice that we have isothermal wall temperature case. The contribution of thermal radiation is modeled using Rosseland approximation. Most of the effort in understanding fluid thermal radiation is devoted to the derivation of reasonable simplification Aboeldahab and ElGendy [17] and Md Miraj et. al. [18] . Rosseland approximation requires that the medium is optically dense and radiation travels only a short distance before being scattered or absorbed. Therefore, a simplified model for Radiative Transfer Equation (RTE) based on Rosseland approximation Md Miraj et. al. [18] is given as

$$
q_{r}=-\frac{4 \sigma^{*}}{3 K^{*}} \frac{\partial T^{4}}{\partial y},
$$

where $\sigma^{*}$ is the stefan-boltzmann constant and $K^{*}$ is known as absorption coefficient. We assumed that the temperature difference within the flow regime are sufficiently small such that $T^{4}$ can be expressed as a linear function of the free stream temperature $T_{\infty}$. This is simplified by expanding $T^{4}$ in taylor series about $T_{\infty}$ and forgone the higher order terms. Considering the Taylor's series expansion of a function $f(x)$ about $x_{0}$

$$
f(x)=f\left(x_{0}\right)+\left(x-x_{0}\right) f^{\prime}\left(x_{0}\right)+\frac{\left(x-x_{0}\right)^{2}}{2 !} f^{\prime \prime}\left(x_{0}\right)+\ldots+\frac{\left(x-x_{0}\right)^{n}}{n !} f^{n}\left(x_{0}\right),
$$

Likewise, expansion of $T^{4}$ about $T_{\infty}$. Setting $f(x)=T^{4}$ and $f\left(x_{0}\right)=f\left(T_{\infty}\right)=T_{\infty}^{4}$ in above equation. Neglecting higher order, we obtain $T^{4}=4 T_{\infty}^{3}-3 T_{\infty}^{4}$. Thus

$$
\frac{1}{\rho c_{p}} \frac{\partial q_{r}}{\partial y}=-\frac{16 \sigma^{*} T_{\infty}^{3}}{3 \rho c_{p} K^{*}} \frac{\partial^{2} T}{\partial y^{2}} .
$$

The pressure imposed on the flow is assumed to be relatively similar to that of the inviscid nonconducting fluid flow about a circular cylinder given as:

$$
\frac{1}{\rho} \frac{\partial P}{\partial x}=\frac{4 u_{\infty}^{2}}{r} \sin \omega \cos \omega .
$$

On applying the boundary layer approximation $(x \gg y)$ and Boussinesq approximation with the statement of facts from equations (1)-(7), the flow model following El-Almin [5] can be given as:

$$
\begin{gathered}
\frac{\partial u}{\partial x}+\frac{\partial v}{\partial y}=0 \\
u \frac{\partial u}{\partial x}+v \frac{\partial u}{\partial y}=\frac{4 u_{\infty}^{2}}{r} \sin \omega \cos \omega+\frac{1}{\rho} \frac{\partial}{\partial y}\left(\mu(T) \frac{\partial u}{\partial y}\right)+g \beta\left(T-T_{\infty}\right) \sin \omega-\frac{\sigma \beta_{0}^{2} u}{\rho_{\infty}}-\frac{\kappa \varepsilon u}{K}-\frac{F \varepsilon^{2} u^{2}}{\sqrt{K}}
\end{gathered}
$$




$$
\begin{aligned}
u \frac{\partial T}{\partial x}+v \frac{\partial T}{\partial y}=\frac{1}{\rho c_{p}} \frac{\partial}{\partial y} & \left(\lambda(T) \frac{\partial T}{\partial y}\right)+\frac{\sigma \beta_{0}^{2} u^{2}}{\rho_{\infty} c_{p}}+\frac{\mu(T)}{\rho C_{p}}\left(\frac{\partial u}{\partial y}\right)^{2} \\
+ & \frac{16 \sigma^{*} T_{\infty}^{3}}{3 \rho c_{p} K^{*}} \frac{\partial^{2} T}{\partial y^{2}}
\end{aligned}
$$

With the boundary conditions:

$$
u(x, 0)=v(x, 0)=0, \quad T(x, 0)=T_{w}, \quad \frac{\partial u(x, \infty)}{\partial y} \rightarrow 0, \quad T(x, \infty) \rightarrow T_{\infty} .
$$

The following relations are introduced to (8)

$$
u=\frac{\partial \psi}{\partial y}, \quad v=-\frac{\partial \psi}{\partial x}
$$

Here, $\psi(x, y)$ represents the stream function and equation (8) is automatically satisfies by (12). Following El-Amin [5], we introduce the following similarity variables and dimensionless temperature

$\theta=\frac{T-T_{\infty}}{T_{w}-T_{\infty}}, \quad \xi(x)=4 \sin ^{2} \frac{w}{2}, \quad \omega=\frac{x}{r}, \quad \eta(x, y)=y \sqrt{\frac{2 u_{\infty}}{\xi \vartheta r}} \sin w, \quad \psi=\sqrt{2 \vartheta r u_{\infty} \xi} f(\xi, \eta)$.

The dimensionless form of viscosity and thermal conductivity variation parameters $D_{\gamma}$ and $D_{\alpha}$ are written as $D_{\gamma}=\gamma\left(T_{w}-T_{\infty}\right)$, and $D_{\alpha}=\alpha\left(T_{w}-T_{\infty}\right)$ respectively. Now substituting (1) - (7) and (13), into equation (8) - (10) we obtain the following

$$
\begin{gathered}
(1+D \gamma-\theta D \gamma) \frac{\partial^{3} f}{\partial \eta^{3}}-D \gamma \frac{\partial \theta}{\partial \eta} \frac{\partial^{2} f}{\partial \eta^{2}}+\frac{\partial^{2} f}{\partial \eta^{2}} f+\left(\left(\frac{2 \xi-4}{4-\xi}\right)+\left(\frac{2 \xi}{\sqrt{4 \xi-\xi^{2}}}\right)\right) k_{2}\left(\frac{\partial f}{\partial \eta}\right)^{2} \\
\pm \frac{N}{4-\xi}+\frac{4-2 \xi}{4-\xi}-\frac{2}{4-\xi}\left(M n+K_{1}\right) \frac{\partial f}{\partial \eta}=2 \xi\left(\frac{\partial f}{\partial \eta} \frac{\partial f^{\prime}}{\partial \xi}-\frac{\partial^{2} f}{\partial \eta^{2}} \frac{\partial f}{\partial \xi}\right) \\
\left(\frac{1}{P r}(1+D \alpha(\theta-1))+\frac{4 R a}{3}\right) \frac{\partial^{2} \theta}{\partial \eta^{2}}+\frac{\partial \theta}{\partial \eta} f-2 n \frac{\partial f}{\partial \eta} \theta+\frac{D \alpha}{P r}\left(\frac{\partial \theta}{\partial \eta}\right)^{2}+2 \xi E c M n\left(\frac{\partial f}{\partial \eta}\right)^{2} \\
+E c \xi(4-\xi)(1+D \gamma-\theta D \gamma)\left(\frac{\partial^{2} f}{\partial \eta^{2}}\right)^{2} \\
=2 \xi\left(\frac{\partial f}{\partial \eta} \frac{\partial \theta}{\partial \xi}-\frac{\partial \theta}{\partial \eta} \frac{\partial f}{\partial \xi}\right)
\end{gathered}
$$

The flow boundary conditions (11) are transformed to

$$
\begin{aligned}
\frac{\partial f(0, \xi)}{\partial \eta}=0, \quad f(0, \xi)+\xi \frac{\partial f(0, \xi)}{\partial \xi} & =0, \quad \theta(0, \xi)=1, \\
\frac{\partial^{2} f(\infty, \xi)}{\partial \eta^{2}} & =0, \quad \theta(\infty, \xi)=0 .
\end{aligned}
$$

It is important to note that in the absence of variable viscosity parameter, thermal conductivity parameter and heat loss by thermal radiation (i.e $D_{\alpha}=D_{\gamma}=R a=0$ ), we obtained the model equations analyzed by El-Amin [5]. 


$$
\begin{gathered}
\text { let } \alpha_{1}=1, \alpha_{2}=D \gamma, \alpha_{3}=\left(\left(\frac{2 \xi-4}{4-\xi}\right)+\left(\frac{2 \xi}{\sqrt{4 \xi-\xi^{2}}}\right)\right) k_{2}, \alpha_{4}=\left(\frac{2 \xi-4}{4-\xi}\right) \alpha_{5}= \pm \frac{N}{4-\xi}, \alpha_{6}= \\
-\frac{2}{4-\xi}\left(M n+K_{1}\right), \alpha_{7}=2 \xi, \alpha_{8}=\frac{1}{P r}, \alpha_{9}=D \alpha, \alpha_{10}=\frac{4 R a}{3}, \alpha_{11}=\frac{D \alpha}{P r}, \alpha_{12}=2 \xi E c M n, \alpha_{13}= \\
E c \xi(4-\xi), \alpha_{14}=-2 n \text {. Hence equations }(14)-(15) \text { become: } \\
\left(\alpha_{1}+\alpha_{2}(1-\theta)\right) f^{\prime \prime \prime}-\alpha_{2} f^{\prime \prime} \theta^{\prime}+\alpha_{1} f^{\prime \prime} f+\alpha_{4}+\alpha_{5} \theta+\alpha_{6} f^{\prime} \alpha_{3} f^{\prime 2} \\
=\alpha_{7}\left(f^{\prime} \frac{\partial f^{\prime}}{\partial \xi}-f^{\prime \prime} \frac{\partial f}{\partial \xi}\right) \\
\left(\alpha_{8}\left(\alpha_{1}+\alpha_{9}(\theta-1)\right)+\alpha_{10}\right) \theta^{\prime \prime}+\alpha_{1} \theta^{\prime} f+\alpha_{14} f^{\prime} \theta+\alpha_{11} \theta^{\prime 2}+\alpha_{12} f^{\prime 2}+\alpha_{13}\left(\alpha_{1}+\alpha_{2}(1-\theta)\right) f^{\prime \prime 2} \\
=\alpha_{7}\left(f^{\prime} \frac{\partial \theta}{\partial \xi}-\theta^{\prime} \frac{\partial f}{\partial \xi}\right) .
\end{gathered}
$$

In practical engineering application ,the physical parameters of practical values are the local skin friction coefficient and local nusselt number of the flow. The wall skin friction and local nusselt number defined are defined as follows:

Skin friction coefficient

$$
C_{f}=4 \sqrt{\frac{2 \sin ^{2} \omega}{\operatorname{Re} \xi}} f^{\prime \prime}(\xi, 0),
$$

Nusselt number

$$
N u=\frac{q_{w}}{T_{w}-T_{\infty}} \frac{r}{k}=-\frac{\sqrt{2} \sin \omega}{\sqrt{\frac{\xi}{R e}}} \theta^{\prime}(\xi, 0) .
$$

\section{Method of solution : BSRM}

In this section, we present the application of bivariate spectral relaxation method (BSRM) to solve the governing coupled non-linear system of partial differential equation (18) - (19). BSRM in its formulation apply Gauss-Siedel relaxation technique to re-arrange and decouple non-linear systems to form a linear sequence of partial differential equations that can be solved in succession over a number of iterations. Consequently, re-arranging equations (18) - (19) and apply the Gauss-Siedel lineariazation scheme, we obtain:

$$
\begin{gathered}
a_{0, r}(\eta, \xi) \frac{\partial^{2} g_{r+1}}{\partial \eta^{2}}+a_{1, r}(\eta, \xi) \frac{\partial g_{r+1}}{\partial \eta}+\alpha_{6} g_{r+1}+a_{2, r}(\eta, \xi) \\
=a_{3, r} \frac{\partial g_{r+1}}{\partial \xi}, \\
\frac{\partial f_{r+1}}{\partial \eta}=g_{r+1}, \\
b_{0, r} \frac{\partial^{2} \theta_{r+1}}{\partial \eta^{2}}+b_{1, r}(\eta, \xi) \frac{\partial \theta_{r+1}}{\partial \eta}+b_{2, r}(\eta, \xi) \theta_{r+1}+b_{3, r}(\eta, \xi) \\
=b_{4, r}(\eta, \xi) \frac{\partial \theta_{r+1}}{\partial \xi},
\end{gathered}
$$

subject to

$$
\begin{aligned}
g_{r+1}(0, \xi)=0, \quad f_{r+1}(0, \xi)+ & 2 \xi \frac{\partial f_{r+1}(0, \xi)}{\partial \xi}=0, \quad \theta_{r+1}(0, \xi)=1, \\
\frac{\partial g_{r+1}(\infty, \xi)}{\partial \eta}=0, \quad \theta_{r+1}(\infty, \xi) & =0,
\end{aligned}
$$


where the coefficients are defined as

$$
\begin{aligned}
& a_{0, r}(\eta, \xi)=\alpha_{1}+\alpha_{2}\left(1-\theta_{r}\right), \quad a_{1, r}(\eta, \xi)=-\alpha_{2} \frac{\partial \theta_{r}}{\partial \eta}+\alpha_{2} f_{r}+\alpha_{7} \frac{\partial \theta_{r}}{\partial \eta} \frac{\partial f_{r}}{\partial \xi} \\
& a_{2, r}(\eta, \xi)=\alpha_{3} g_{r}^{2}+\alpha_{4}+\alpha_{5} \theta_{r}, \quad a_{3, r}(\eta, \xi)=\alpha_{7} g_{r}, \\
& b_{0, r}(\eta, \xi)=\alpha_{8} \alpha_{1}-\alpha_{8} \alpha_{9}+\alpha_{8} \alpha_{10}+\alpha_{8} \alpha_{9} \theta_{r}, \quad b_{1, r}(\eta, \xi)=\alpha_{1} f_{r}+\alpha_{7} \frac{\partial \theta_{r}}{\partial \eta} \frac{\partial f_{r}}{\partial \xi}, \\
& b_{2, r}(\eta, \xi)=\alpha_{13} \alpha_{1}\left(\frac{\partial \theta_{r}}{\partial \eta}\right)^{2}+\alpha_{13} \alpha_{2}\left(\frac{\partial \theta_{r}}{\partial \eta}\right)^{2}-\alpha_{13} \alpha_{2} g_{r}^{2}+\alpha_{4} g_{r}, \quad b_{3, r}(\eta, \xi)=\alpha_{11} \theta_{r}^{2}+\alpha_{2} g_{r}^{2}, \\
& b_{4, r}(\eta, \xi)=\alpha_{7} g_{r} .
\end{aligned}
$$

The set of equations (20) -(24) are solve iteratively starting from $r=1,2, \ldots$, and using a given initial approximations, denoted here as $g_{0}, f_{0}$ and $\theta_{0}$ until approximate solutions are obtained that is consistent with a given tolerance level. By adopting the similar procedures as stated in a related paper by Motsa et al. [26], spectral collocation method can be used to discretize the independent variables $\eta$ and $\xi$ domains. The Chebyshev collocation technique as applied in spectral collocation methods requires that the domain of the problem be transformed from $[0, \infty]$ to $[-1,1]$. Hence, using linear transformation, we transform $\eta \in\left[0, \eta_{\infty}\right]$ and $\xi \in[0,1]$ to $\tau \in[-1,1]$ and $\zeta \in[-1,1]$, respectively. Here $\eta_{\infty}$ is a finite value that is introduced to facilitate the application of the numerical method at infinity. The spatial and time domains are disctretized using Chebyshev Gauss-Lobatto points defined as

$$
\tau_{i}=\cos \left(\frac{\pi i}{N_{x}}\right), \quad \zeta_{j}=\cos \left(\frac{\pi j}{N_{t}}\right), \quad i=0,1, \ldots, N_{x} ; j=0,1, \ldots, N_{t}
$$

Considering that the linear system of PDEs (20) - (22) is decoupled, each equation can be solved independently of the other equations in the system. For example, in solving (20) it is assumed that the solution for $g(\eta, \xi)$ can be approximated by a bivariate Lagrange interpolation polynomial of the form:

$$
g(\eta, \xi) \approx \sum_{m=0}^{N_{x}} \sum_{j=0}^{N_{t}} g\left(\tau_{m}, \zeta_{j}\right) L_{m}(\tau) L_{j}(\zeta)
$$

which interpolates $g(\eta, \xi)$ at the collocation points defined by equation (22). We remark that, for ease of notation, we have dropped the subscripts $r+1$. The functions $L_{m}(\tau)$ are the well-known characteristic Lagrange cardinal polynomials

$$
L_{m}(\tau)=\prod_{\substack{m=0 \\ m \neq k}}^{N_{x}} \frac{\tau-\tau_{k}}{\tau_{m}-\tau_{k}}, \quad L_{m}\left(\tau_{k}\right)=\delta_{m k}= \begin{cases}0 & \text { if } m \neq k \\ 1 & \text { if } m=k\end{cases}
$$

The function $L_{j}(\zeta)$ is defined in a similar manner. Equation (26) is then substituted in equation (20) and (21). A key step in the substitution process is the evaluation of the derivatives of $L_{m}(\tau)$ and $L_{j}(\zeta)$ with respect to $\tau$ and $\zeta$ respectively. Several formulas exist for computing the derivatives when the collocating points are chosen to be Chebyshev Gauss-Lobatto points of the form (25). The method of discretization of PDEs using the bivariate approach described above has also been used in [30] who solved one equation of PDEs for different models of non-linear evolution parabolic PDEs.

Following [31,32], we define the derivatives of $g(\eta, \xi)$ with respect to $\eta$ and $\xi$ at the collocating points $\tau_{k}$ and $\zeta_{i}$ as follows: 


$$
\begin{aligned}
\left.\frac{\partial g}{\partial \eta}\right|_{\left(\tau_{k}, \zeta_{i}\right)} & =\frac{2}{\eta_{\infty}} \sum_{m=0}^{N_{x}} \sum_{j=0}^{N_{t}} g\left(\tau_{m}, \zeta_{j}\right) \frac{d L_{m}\left(\tau_{k}\right)}{d \tau} L_{j}\left(\zeta_{i}\right)=\mathbf{D G}_{i}, \\
\left.\frac{\partial^{2} g}{\partial \eta^{2}}\right|_{\left(\tau_{k}, \zeta_{i}\right)} & =\mathbf{D}^{2} \mathbf{G}_{i}, \\
\left.\frac{\partial g}{\partial \xi}\right|_{\left(\tau_{k}, \zeta_{i}\right)} & =2 \sum_{m=0}^{N_{x}} \sum_{j=0}^{N_{t}} g\left(\tau_{m}, \zeta_{j}\right) \frac{d L_{j}\left(\zeta_{i}\right)}{d \zeta} L_{m}\left(\tau_{k}\right)=2 \sum_{j=0}^{N_{t}} d_{i j} \mathbf{G}_{j},
\end{aligned}
$$

here $d_{i, j}\left(i, j=0,1, \ldots, N_{t}\right)$ are values of the standard Chebyshev differentiation matrix $d=\left[d_{i, j}\right]$ of size $\left(N_{t}+1\right) \times\left(N_{t}+1\right)$ (see, for example [31,32]), $\mathbf{D}=\left(2 / \eta_{e}\right)\left[D_{r, s}\right]\left(r, s=0,1,2, \ldots, N_{x}\right)$ with $\left[D_{r, s}\right]$ being an $\left(N_{x}+1\right) \times\left(N_{x}+1\right)$ Chebyshev derivative matrix, and the vector $\mathbf{G}_{i}$ is defined as

$$
\mathbf{G}_{i}=\left[g_{i}\left(\tau_{0}\right), g_{i}\left(\tau_{1}\right), \ldots, g_{i}\left(\tau_{N_{x}}\right)\right]^{T} .
$$

Applying the collocation method with the above definitions on equations (20) gives

$$
\mathbf{A}_{i} \mathbf{G}_{r+1, i}+\mathbf{a}_{2, r}\left(\xi_{i}\right)-2 \mathbf{a}_{3, r}\left(\xi_{i}\right) \sum_{j=0}^{N_{t}} d_{i, j} \mathbf{G}_{r+1, j}=0, \quad i=0,1,2, \ldots, N_{t},
$$

subject to the boundary conditions:

$$
g_{r+1, i}\left(\tau_{N_{x}}\right)=0, \quad \sum_{i=0}^{N_{t}} \mathbf{D g}_{r+1, i}=0
$$

where

$$
\mathbf{A}_{i}=\mathbf{a}_{0, r}\left(\xi_{i}\right) \mathbf{D}^{2}+\mathbf{a}_{1, r}\left(\xi_{i}\right) \mathbf{D}+\alpha_{6} \mathbf{I},
$$

$\mathbf{a}_{m, r}\left(\xi_{i}\right) \quad(m=0,3)$ is the diagonal matrix of size $\left(N_{x}+1\right) \times 1$ of vector form $\left[a_{m, r}\left(\tau_{0}\right), a_{m, r}\left(\tau_{1}\right), \ldots, a_{m, r}\left(\tau_{N_{x}}\right)\right]^{T}$.

Expanding equation (32) and imposing boundary conditions for $i=0,1, \ldots, N_{t}$ gives the following matrix equation:

$$
\left[\begin{array}{cccc}
A_{0,0} & A_{0,1} & \cdots & A_{0, N_{t}} \\
A_{1,0} & A_{1,1} & \cdots & A_{1, N_{t}} \\
\vdots & \vdots & \ddots & \vdots \\
A_{N_{t}, 0} & A_{N_{t}, 1} & \cdots & A_{N_{t}, N_{t}}
\end{array}\right]\left[\begin{array}{c}
\mathbf{G}_{r+1,0} \\
\mathbf{G}_{r+1,1} \\
\vdots \\
\mathbf{G}_{r+1, N_{t}}
\end{array}\right]=\left[\begin{array}{c}
\mathbf{R}_{1,0} \\
\mathbf{R}_{1,1} \\
\vdots \\
\mathbf{R}_{1, N_{t}}
\end{array}\right]
$$

where

$$
\begin{aligned}
& A_{i, i}=\mathbf{A}_{i}-2 \mathbf{a}_{3, r}\left(\xi_{i}\right) d_{i, i} \mathbf{I}, \quad i=0,1, \ldots, N_{t}-1, \\
& A_{i, j}=-2 \mathbf{a}_{3, r}\left(\xi_{i}\right) d_{i, i} \mathbf{I}, \quad \text { when } i \neq j, \\
& \mathbf{R}_{1, i}=-\mathbf{a}_{2, r}\left(\xi_{i}\right),
\end{aligned}
$$

where $\mathbf{I}$ is an $\left(N_{x}+1\right) \times\left(N_{x}+1\right)$ identity matrix.

Similarly, applying the bivariate collocation as described above on equations (22) gives

$$
\mathbf{B}_{i} \Theta_{r+1, i}+\mathbf{b}_{3, r}\left(\xi_{i}\right)
$$




$$
-2 \mathbf{b}_{4, r}\left(\xi_{i}\right) \sum_{j=0}^{N_{t}} d_{i, j} \Theta_{r+1, j}=0, \quad i=0,1,2, \ldots, N_{t},
$$

under the boundary conditions:

$$
\theta_{r+1, i}\left(\tau_{N_{x}}\right)=1, \quad \theta_{r+1, i}\left(\tau_{0}\right)=0
$$

where

$$
\begin{aligned}
\mathbf{B}_{i} & =\mathbf{b}_{0, r}\left(\xi_{i}\right) \mathbf{D}^{2}+\mathbf{b}_{1, r}\left(\xi_{i}\right) \mathbf{D}+\mathbf{b}_{2, r}\left(\xi_{i}\right), \\
\Theta_{i} & =\left[\theta_{i}\left(\tau_{0}\right), \theta_{i}\left(\tau_{1}\right), \ldots, \theta_{i}\left(\tau_{N_{x}}\right)\right]^{T},
\end{aligned}
$$

$\mathbf{b}_{m, r}\left(\xi_{i}\right)(m=0,4)$ are the diagonal matrix of size $\left(N_{x}+1\right) \times 1$ of the vector form $\left[b_{m, r}\left(\tau_{0}\right), b_{m, r}\left(\tau_{1}\right), \ldots, b_{m, r}\left(\tau_{N_{x}}\right)\right]^{T}$.

Expanding equations (38) and imposing the boundary conditions for $i=0,1, \ldots, N_{t}-1$ gives the following matrix equations:

$$
\left[\begin{array}{cccc}
B_{0,0} & B_{0,1} & \cdots & B_{0, N_{t}} \\
B_{1,0} & B_{1,1} & \cdots & B_{1, N_{t}} \\
\vdots & \vdots & \ddots & \vdots \\
B_{N_{t}, 0} & B_{N_{t}, 1} & \cdots & B_{N_{t}, N_{t}}
\end{array}\right]\left[\begin{array}{c}
\Theta_{r+1,0} \\
\Theta_{r+1,1} \\
\vdots \\
\Theta_{r+1, N_{t}}
\end{array}\right]=\left[\begin{array}{c}
\mathbf{R}_{2,0} \\
\mathbf{R}_{2,1} \\
\vdots \\
\mathbf{R}_{2, N_{t}}
\end{array}\right]
$$

where

$$
\begin{aligned}
B_{i, i} & =\mathbf{B}_{i}-2 \mathbf{b}_{4, r}\left(\xi_{i}\right) d_{i, i} \mathbf{I}, \quad i=0,1, \ldots, N_{t}, \\
B_{i, j} & =-2 \mathbf{b}_{4, r}\left(\xi_{i}\right) d_{i, i} \mathbf{I}, \quad \text { when } i \neq j, \\
\mathbf{R}_{2, i} & =-\mathbf{b}_{3, r}\left(\xi_{i}\right),
\end{aligned}
$$

In order to obtain an approximate solutions for the unknown functionals as described above,we set the initial approximations as

$$
f_{0}(\eta, \xi)=1-e^{-\eta^{2}}, \quad \theta_{0}(\eta, \xi)=e^{-2 \eta},
$$

with a view to satisfy with the boundary conditions (23)- (24)

Hence, starting from the initial approximations $f_{0}(\eta, \xi)$ and $\theta_{0}(\eta \cdot \xi)$ as given by equation ((44)), An approximate solutions for $f(\eta, \xi), g(\eta, \xi)$ and $\theta(\eta, \xi)$ are derived by iteratively solving the matrix equations ((34)) and ((40)), in turn, for $r=0,1,2, \ldots$ until a solution is obtained that converges to a given level of accuracy.

\section{Results and Discussion}

The governing system of equations (14) - (15) subject to (16) have been solved using the bivariate spectral relaxation method (BSRM) as described previously. In this section, we present the numerical computations for velocity, temperature, skin friction coefficient and local heat transfer rate profiles for the various pertinent parameters. For purpose of discussing our results, an important parameters such as $(D \gamma, D \alpha, R a, N, M n$, and $\xi)$ have been varied at a fixed values of $k 2=0.05, k 1=0.5, R a=$ $0.5 ; E c=0.01, n=1,=0.0$ to obtain a clear insight into the physics of the problem. In computing the numerical results presented in this paper, unless otherwise stated, the following values of physical parameters were used : $D \gamma=0.5, \operatorname{Pr}=0.7, \xi=0.0, D \alpha=0.1, \omega=18^{\circ}, M n=0.5$ and $N=2$. Grid independence tests revealed that at collocation points of $N_{x}=80$ and $N_{t}=1.5$ in the $\eta$ and $\xi$ domain 
respectively, an accurate and consistent results were attained with a few iteration. A further increase in the number of collocation points did not result in a change in the computed results. Furthermore, the minimum number of iterations required to give results that are consistent within a tolerance level of $10^{-7}$ were used. In all the results presented below, it was found that 60 iterations were sufficient to give consistent results. The value of $\eta_{\infty}$ was set to be 10 .

Table 1: Comparison of present study with El- Almin [5] on the variation of wall shear stress $f^{\prime \prime}(\xi, 0)$ and local heat transfer rate $-\theta^{\prime}(\xi, 0)$ when $D \gamma=0, D \alpha=0, R=0$.

\begin{tabular}{|c|c|c|c|c|c|c|}
\hline \multirow{2}{*}{$\mathrm{Mn}$} & \multirow{2}{*}{$\mathrm{k} 1$} & \multirow{2}{*}{$\mathrm{k} 2$} & \multicolumn{2}{|c|}{ El-Amin[5] } & \multicolumn{2}{|c|}{ Present study } \\
\hline & & & $f^{\prime \prime}(\xi, 0)$ & $-\theta^{\prime}(\xi, 0)$ & $f^{\prime \prime}(\xi, 0)$ & $-\theta^{\prime}(\xi, 0)$ \\
\hline \multirow[t]{9}{*}{0.0} & 0 & 0 & 2.9389 & 0.5932 & 2.9342 & 0.5983 \\
\hline & & 0.5 & 2.7050 & 0.5527 & 2.7088 & 0.5542 \\
\hline & & 1 & 2.5091 & 0.5211 & 2.5135 & 0.5245 \\
\hline & 0.5 & 0 & 2.6322 & 0.5516 & 2.6346 & 0.5513 \\
\hline & & 0.5 & 2.4279 & 0.5147 & 2.4349 & 0.5149 \\
\hline & & 1 & 2.2876 & 0.4896 & 2.2790 & 0.4861 \\
\hline & 1 & 0 & 2.3400 & 0.5054 & 2.3317 & 0.5146 \\
\hline & & 0.5 & 2.2036 & 0.4791 & 2.2096 & 0.4713 \\
\hline & & 1 & 2.1057 & 0.4607 & 2.1021 & 0.4616 \\
\hline \multirow[t]{9}{*}{0.5} & 0 & 0 & 2.7929 & 0.5664 & 2.7458 & 0.5607 \\
\hline & & 0.5 & 2.5661 & 0.5267 & 2.5349 & 0.5248 \\
\hline & & 1 & 2.3978 & 0.4991 & 2.3872 & 0.4968 \\
\hline & 0.5 & 0 & 2.4855 & 0.5216 & 2.4709 & 0.5146 \\
\hline & & 0.5 & 2.314 & 0.4907 & 2.3096 & 0.4877 \\
\hline & & 1 & 2.1958 & 0.4699 & 2.1821 & 0.4627 \\
\hline & 1 & 0 & 2.2244 & 0.4795 & 2.2299 & 0.4793 \\
\hline & & 0.5 & 2.1132 & 0.459 & 2.1930 & 0.4533 \\
\hline & & 1 & 2.0231 & 0.4513 & 1.9875 & 0.4438 \\
\hline
\end{tabular}

In order to test our method of solution, we first solve special case of our problem which are also compared with El- Amin [5] in Table 1. Table 1 is drawn for a special case when pertinent parameters $D \alpha=D \gamma=R=0$. The available results obtained in [5] and the present results are compared. The comparison shows that the present results obtained using BSRM were in excellent agreement with the solution of El-Amin [5]. The results from the table 1 are consistent with the observations made in Fig. 5 where the wall shear stress and local heat transfer are observed to decrease with an increase in $M n$. This is because the magnetic force and the medium pores has the capacity to retard the fluid flow in conjunction with the adverse pressure to resist the flow. The magnetic force and porous medium pores work against the inertial force and this consequently reduce the absolute value of the local heat transfer. The velocity gradient at the wall surface $f^{\prime \prime}(\xi, 0)$ that is, the wall shear stress decreases with increase in magnetic parameter $\mathrm{Mn}$. From the Table 1, we noticed that an increase in the values of magnetic force $\mathrm{Mn}$ on the flow regime results to the narrowing of the horizontal velocity of the fluid. The transverse contraction on the velocity boundary layer of the flow is due to the applied magnetic field, which consequently invokes lorentz force producing noticeable opposition to the flow of the fluid. Hence, the magnetic parameter Mn influences the control of surface shear stress.

Table 2 gives account of the effect of viscous variation parameter $D \gamma$, thermal conductivity variation parameter $D \alpha$ and thermal radiation parameter $\mathrm{R}$ on the local heat transfer rate. Consequently, It is observe that an increase in thermal radiation parameter $\mathrm{R}$, viscous variation parameter $D \gamma$ and 
Table 2: Computed values of wall shear stress $f^{\prime \prime}(\xi, 0)$ and local heat tranfer rate $-\theta^{\prime}(\xi, 0)$ for different values of $D \gamma, D \alpha$ and $R a$ when $P r=0.7, n=0.0, N=10.0, E c=0.01, k 2=0.5, k 1=0.05$, $\omega=30 \circ, M n=0.0$

\begin{tabular}{llll}
\hline $\mathrm{Ra}$ & $D \gamma$ & $D \alpha$ & $-\theta^{\prime}(\xi, 0)$ \\
\hline 0.0 & 0 & 0 & 0.5466 \\
& & 0.5 & 0.5305 \\
& 0.5 & 1 & 0.5179 \\
\hline & & 0 & -0.0322 \\
& 0 & 0.5 & -0.0572 \\
& & 1 & -0.0766 \\
\hline 0.5 & & 0 & 0.4624 \\
& 0.5 & 0.5 & 0.4488 \\
& & 1 & 0.4381 \\
\hline 1.0 & 0 & 0.5 & 0.1403 \\
& 0 & 1 & 0.1213 \\
& & 0 & 0.1065 \\
\hline & & 0.5 & 0.4081 \\
& 0.5 & 1 & 0.3964 \\
& & 0 & 0.3872 \\
\hline
\end{tabular}



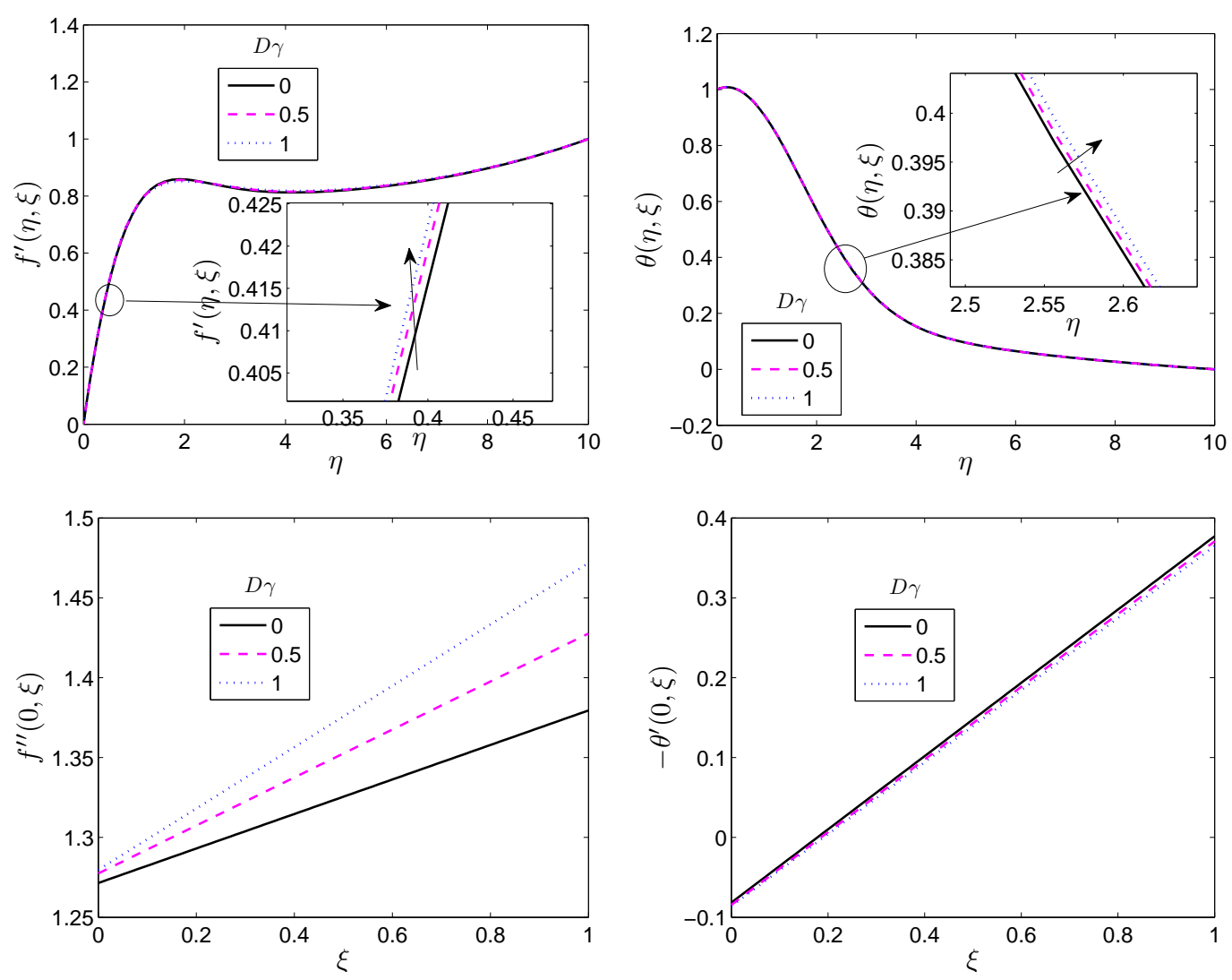

Fig. 1: Effect of viscous variation parameter $(D \gamma)$ on velocity,temperature,skin friction coefficient and local heat transfer rate profiles
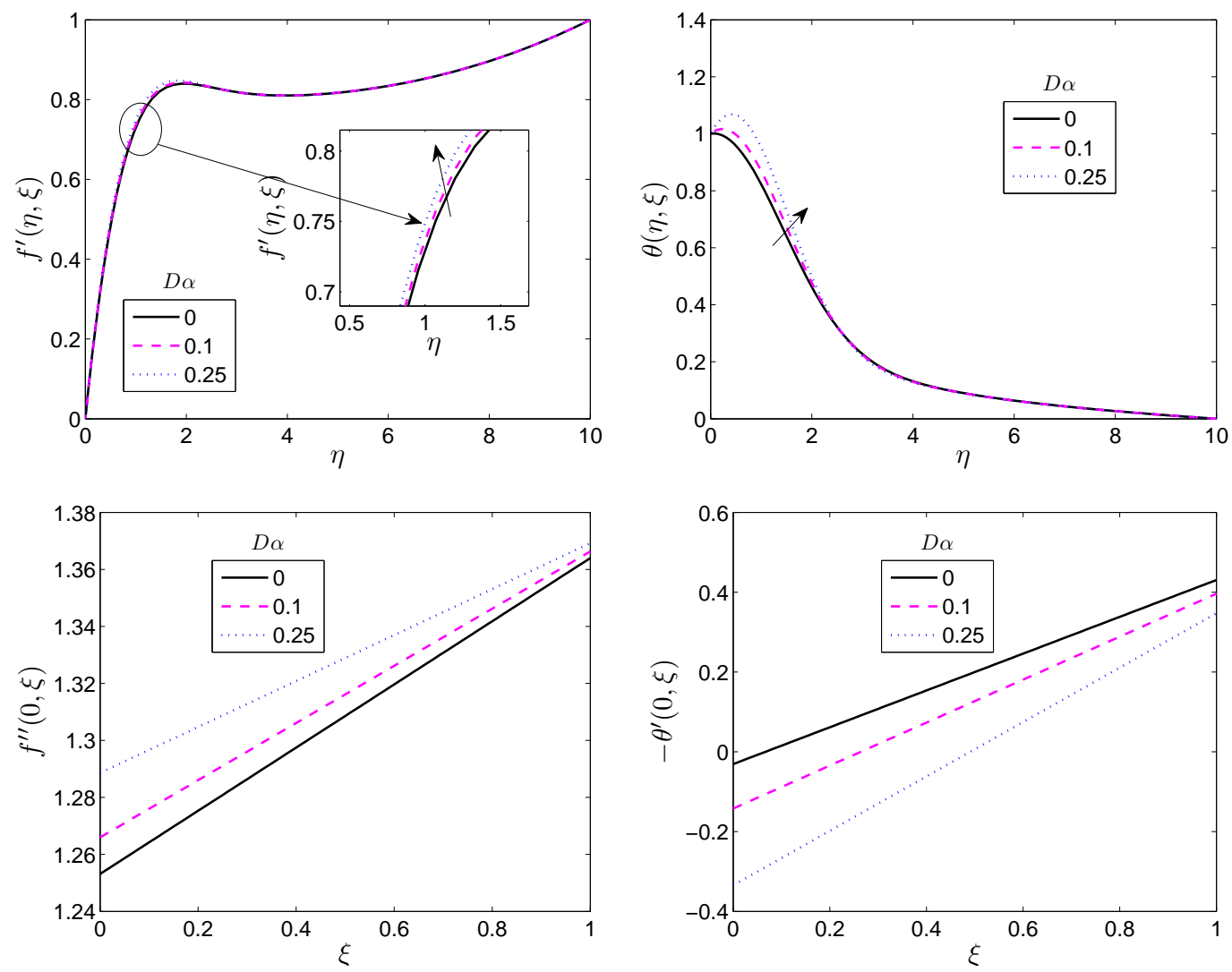

Fig. 2: Effect of thermal conductivity variation parameter $(D \alpha)$ on velocity, temperature, skin friction coefficient and local heat transfer rate profiles 

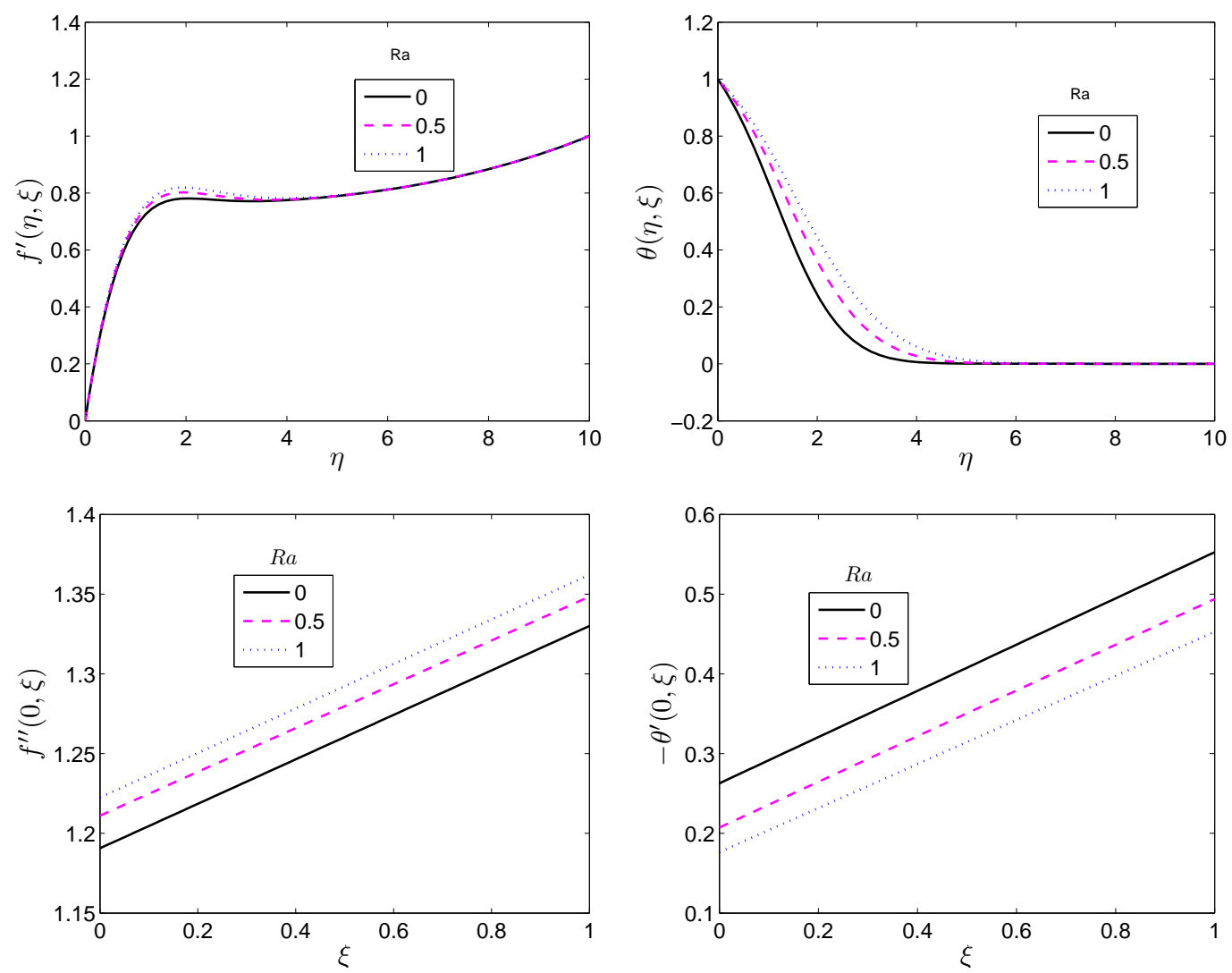

Fig. 3: Effect of thermal radiation $(R)$ on velocity, temperature, skin friction coefficient and local heat transfer rate profiles
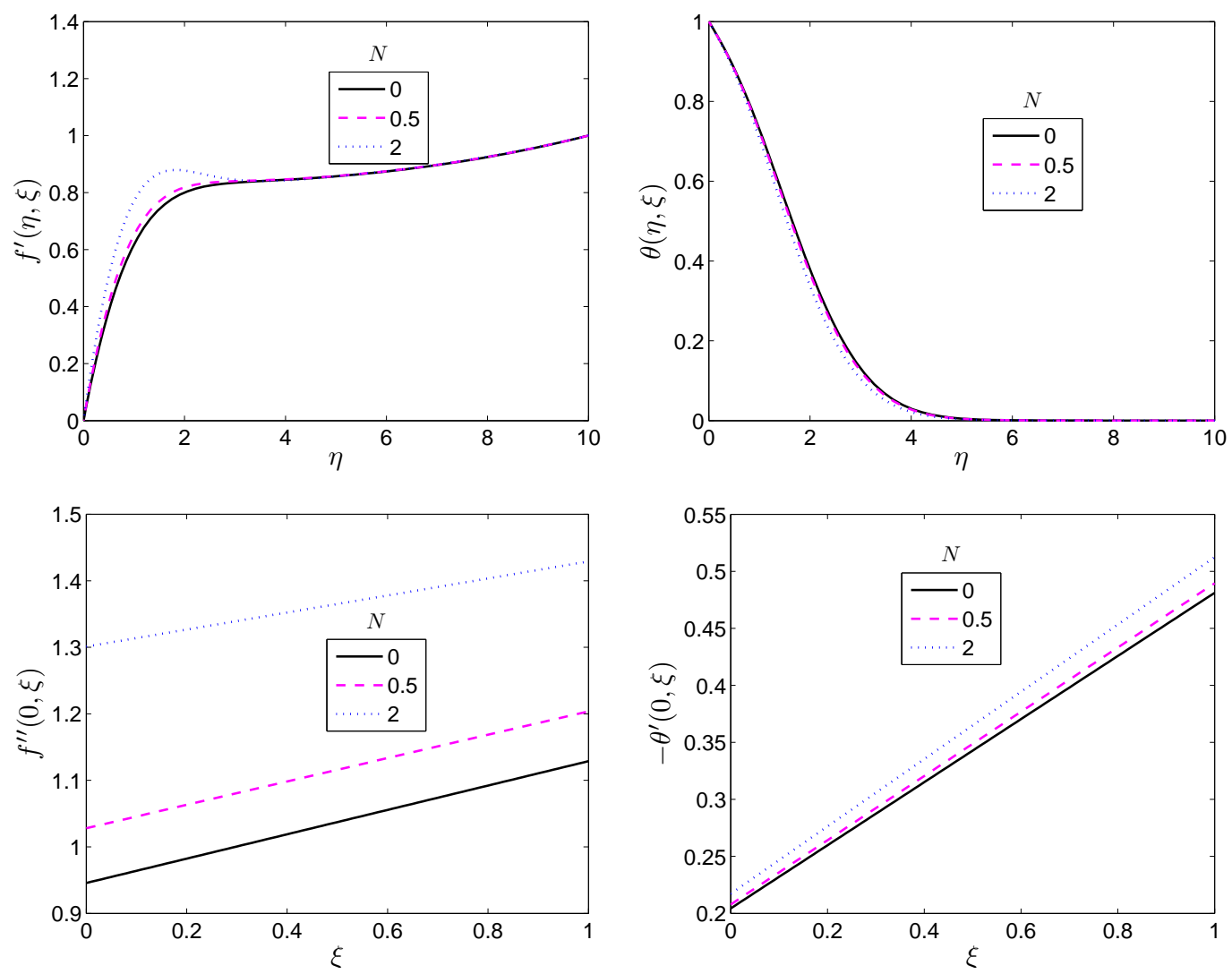

Fig. 4: Effect of Bouyancy parameter $(N)$ on velocity, temperature, skin friction coefficient and local heat transfer rate profiles 

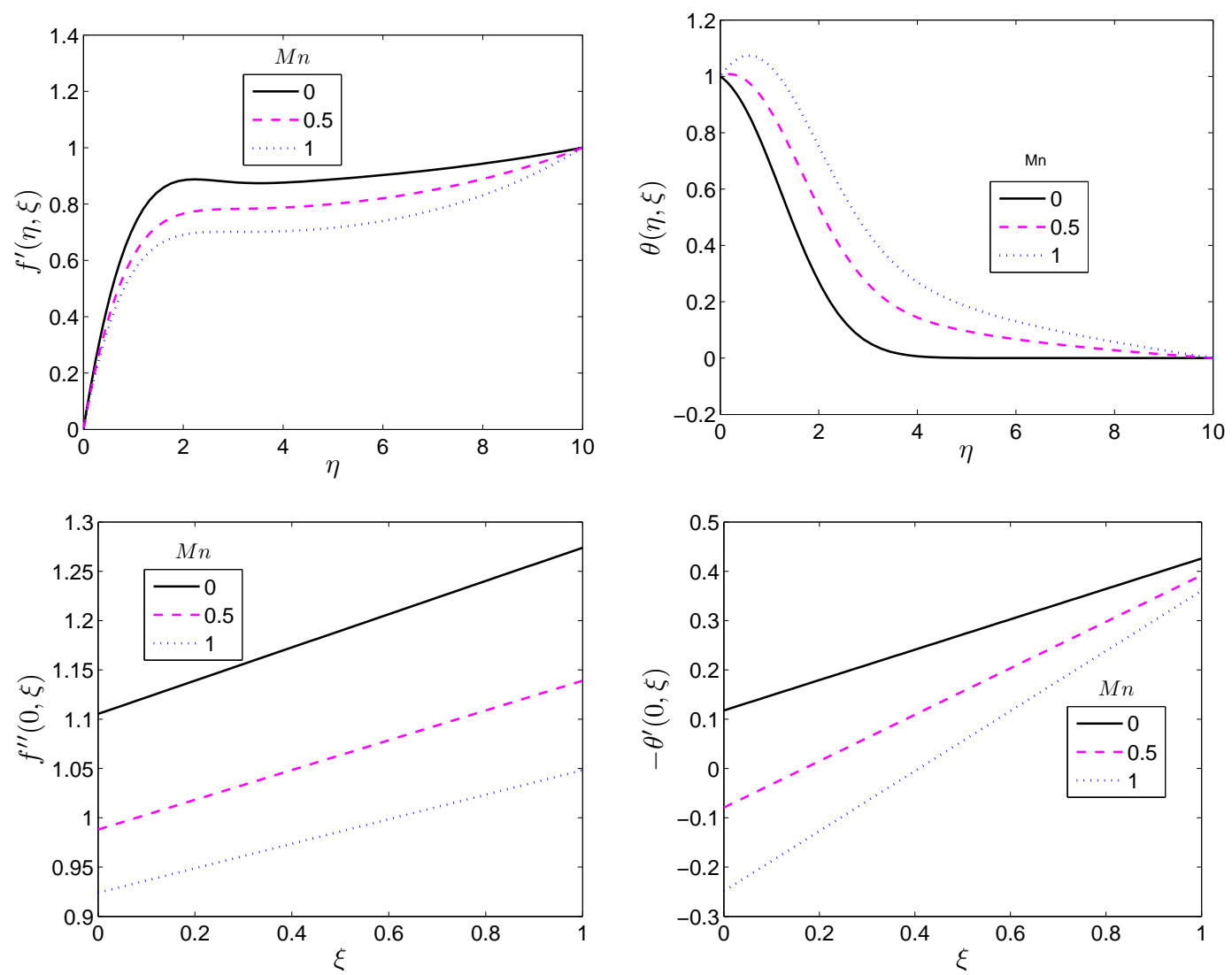

Fig. 5: Effect of magnetic parameter $(M n)$ on velocity, temperature, skin friction coefficient and local heat transfer rate profiles

thermal conductivity variation parameter $D \alpha$ of the viscous fluid causes a notable decrease in local heat energy transfer within the flow regime. In Fig.Fig. 1, we present variation of viscous parameter $(D \gamma)$ and its effects on the fluid velocity, temperature, skin friction and local heat transfer. We observe that increase in viscous parameter cause a slight significant increment in the fluid velocity, temperature and local heat transfer respectively, whereas a remarkable increment is notice in the skin friction. Figure 2 presents variation of thermal conductivity parameter $(D \alpha)$ and its effect on the fluid velocity, temperature, skin friction and local heat transfer. It is obvious from Fig. 2 that increase in thermal conductivity parameter cause slight increment in the fluid velocity and temperature whereas significant increment and decrement are observed in skin friction and local heat transfer respectively. Figure 3 gives account of variation of thermal radiation parameter $(R)$ over the fluid velocity, temperature, skin friction and local heat transfer. It is apparent that noticeable increment in fluid velocity and temperature are observed as thermal radiation parameter increases whereas remarkable increment and reduction are noticed in skin friction and local heat transfer respectively. The results qualitatively agree with the report from literature that one of the tangible effect of thermal radiation and surface temperature on flow is to enhance the rate of heat energy transfer. Hence, one can easily control the rate of heat transfer characteristics using thermal radiation mechanism.

The flow profiles for various values of buoyancy parameter $\mathrm{N}$ is shown in Fig. 4. It is obvious that increment in Buoyancy parameter induces $r=$ the fluid velocity and temperature to increase and decrease respectively, whereas we notice a significant increment in skin friction and local heat transfer respectively. Fig. 5, displays the influence of magnetic field force on fluid velocity, temperature, momentum boundary layer profile as well as the local heat transfer rate profile. We notice remarkable reduction and increment in fluid velocity and temperature as magnetic parameter increases respectively, whereas significant reduction is observed in skin friction and local heat transfer. It is remarkable to point that magnetic force contribute to the damping effect on the flow velocity field by creating drag 
force which opposes the fluid motion thereby causing reduction in the velocity of the flow. This indicates that transverse magnetic field on the flow opposes the transport phenomena. More importantly variation of magnetic parameter $M n$ corresponds to variation of lorentz force due to the applied magnetic field, thereby offers more resistance to the transport phenomena. Hence, momentum boundary layer thickness becomes larger and the separation of boundary layer is attained early. The effect of Lorentz force on velocity profiles generated a kind of friction on the flow; this friction in turn generated more heat energy which eventually increases the temperature distribution in the flow . That is, increasing the magnetic force on the flow regime has positive impact on the temperature distribution in the flow flow and thereby decreases the wall gradient. Meanwhile, its increases the thickness of the thermal boundary layer.

\section{Conclusion}

Magnetohydrodynamic forced convective flow over a non-isothermal horizontal circular cylinder embedded in a fluid saturated porous medium has been investigated with the consideration of the effects of variable viscosity and thermal conductivity in the presence of thermal radiation. The viscosity and thermal conductivity of the working fluid have been modeled as a linear function of temperature. Rosseland diffusion approximation have been used to describe and model the radiative heat loss on the flow. In this paper, a new spectral collocation based method expressed in terms of bivariate Lagrange interpolation polynomials and adapted to coupled non-linear systems of partial differential equations using relaxation techniques has been applied. The derivation of the method was found to be straightforward because it does not depend on any linearisation expansions and the discretization of the ordinary and partial derivatives was based on simple formulas. The influence of various physical parameters such as varuable viscosity $D \gamma$, thermal conductivity parameter $D \alpha$, thermal radiation $R a$ and magnetic parameter $M n$ were also investigated and analyzed. The results from this study indicate that variable viscosity and thermal conductivity have strong influence on the flow field regime. Other parameters which appear to have a marginal influence on the velocity and temperature profiles also have strong impact on the surface shear stress and local heat transfer rate. Comparison with previous study was performed and the results were found to be in good agreement. Our findings reveal that:

$\diamond$ An increase in the magnitude of viscous variation parameter $D \gamma$ and thermal conductivity variation parameter $D \alpha$ of the working fluid increases the flow velocity and temperature distribution in the boundary layer region.

$\diamond$ The variation of thermal radiation parameter shows a significant influence on the proportion of boundary layer thickness. (i. e. velocity and temperature profiles). An increase in thermal radiation parameter $\mathrm{R}$ lead to a significant increase in both axial velocity and temperature profiles within the boundary layer region but lower the local heat transfer rate.

$\diamond$ Increasing the magnetic field strength of the magnetohydrodynamics forced convective flow brings about reduction in the wall shear stress and the local heat transfer rate whereas, there is corresponding increase in the velocity and temperature profiles. Hence, using magnetic field force one can easily control the flow direction and the amount of heat transfer characteristics.

$\diamond$ The results obtained in this investigation have clearly shown that the assumption of constant values case for the fluid properties may contribute severe errors when predicting and modeling the proportion of surface shear stress and rate of heat transfer in transport phenomena . 


\section{Nomenclature}

\section{English}

symbols

$B_{1} \quad$ Difference in the temperature between the wall and the flow stream temperature $\left(T_{w}-T_{\infty}\right)$

$c_{p} \quad$ The specific heat at constant pressure

$D \alpha \quad$ Thermal conductivity variation parameter $\left(\alpha B_{1}\right)$

$D \gamma \quad$ Viscous variation parameter $\left(\gamma B_{1}\right)$

Ec Eckert number $\left(\frac{u_{\infty}^{2}}{B_{1} c_{p}}\right)$

F Empirical constant

$g \quad$ Acceleration due to gravity

Gr Grashof mass number $\left(\frac{g \beta\left(T_{w}-T_{\infty}\right) r^{3} \rho^{2}}{k}\right)$

$H a$ Hartmann number $\left(\beta_{0} r \sqrt{\frac{\sigma}{k}}\right)$

$k 1 \quad$ First order solid matrix resistance $\left(\frac{r \varepsilon \vartheta}{K u_{\infty}}\right)$

$k 2 \quad$ Second order solid matrix resistance parameter $\left(\frac{F r \varepsilon^{2}}{\sqrt{K}}\right)$

$K \quad$ Permeability

$P \quad$ Pressure of the fluid

$M n \quad$ Magnetic parameter $\left(\frac{H a^{2}}{R e}\right)$

$N \quad$ Buoyancy parameter $\left(\frac{G r}{R e^{2}}\right)$

$\operatorname{Pr} \quad$ Prandtl number $\left(\frac{\rho_{\infty \vartheta}}{\lambda_{\infty}}\right)$

$q_{r} \quad$ Radiative heat flux

Re Reynold number $\left(\frac{u_{\infty} r \rho}{\vartheta}\right)$

$R a \quad$ Thermal radiation parameter $\left(\frac{4 \sigma^{*} T_{\infty}^{3}}{\vartheta K^{*}}\right)$

$T_{\infty} \quad$ Free stream temperature of the working fluid

$\mathrm{u}, \mathrm{v} \quad$ Velocity component in $\mathrm{x}$ - and $\mathrm{y}$ - direction respectively

$C_{f} \quad$ Skin- friction coefficient

$f \quad$ Dimensionlesss stream function

$\mathrm{Nu} \quad$ Nusselt number 


$\begin{array}{ll}\text { Greek symbols } \\ \mu & \text { Dynamic viscosity } \\ \rho & \text { Density of the fluid } \\ \vartheta & \text { kinematic viscosity } \\ \alpha & \text { Thermal diffusivity term } \\ \beta_{0} & \text { Magnetic field of constant strenght } \\ \sigma * & \text { Electrical conductivity } \\ \tau_{w} & \text { Wall shear stress } \\ \eta & \text { Similarity variable } \\ \psi & \text { Stream function } \\ \beta & \text { Thermal expansion coefficient } \\ \lambda & \text { Thermal conductivity } \\ \omega & \text { Angular velocity } \\ \varepsilon & \text { Porosity } \\ \theta & \text { Dimensionless temperature function } \\ \rho_{\infty} & \text { Ambient density of the working fluid }\end{array}$

\section{References}

[1] K. Vafai, Handbook od porous media, 2nd edition, Taylor and Francis and CRC press, Boca Raton, 2005.

[2] D.A. Nield, A. Bejan, Convection in porous media, 3rd edition, Springer-Verlag, New-York, 2006.

[3] D.B. Ingham, I. Pop, Transport phenomena in porous media III. Vol. 3, Elsevier, London, 2005.

[4] I. Pop, D.B. Ingham, Convective heat transfer: Mathematical and Computational modelling of viscous fluids and porous median, Pergamon, Oxford, 2001.

[5] M.F. El-Amin, Combined effect of viscous dissipation and joule heating on MHD forced convection over a non-isothermal horizontal cylinder embedded in a fluid saturated porous medium, Journal of Magnetism and Magnetic Materials. 263(3) (2003) 337-343.

[6] D. Srinivasacharya, J. Pranitha, Ch. RamReddy, Magnetic effect on free convection in a nondaryc porous medium saturated with doubly stratifield power-law fluid, J. Braz. Soc. Mech. Sci. and Eng. 33(1) (2011) 8-14.

[7] Cheng Ching-Yang, Combined heat and mass transfer in natural convection flow from a verticla wavy surface in power-law fluid saturated porous medium with thermal and mass stratification, Int. Comm. Heat Mass Transfer. 36 (2009) 351-356.

[8] S. Mukhopadhyay, Effect of thermal radiation on unsteady mixed convection flow and heat transfer over a porous stretching surface in porous medium, Int. J. Heat and Mass Transfer. 52(13-14) (2009) 3261-3265.

[9] A. Pantokratoras, Furhter results on variable viscosity on flow and heat transfer to a continuos moving flat plate, Int. J Engineering Sciences. 42 (2004) 1891-1896. 
[10] K. S. Adegbie, F.I. Alao, Flow of temperature dependent viscous fluid between parallel heated walls: Exact analytical solutions in the presesnce of viscous dissipation, Journal of Mathematics and Statistics. 3(1) (2007) 12-14.

[11] S. Ahmad et al., Mixed convection boundary layer flow past an isothermal horizontal circular cylinder with temperature-dependent viscosity, Int. J. of Thermal Sciences. 48 (2009) 1943-1948.

[12] P. Ganesan, G. Palani, Finite difference analysis of unsteady natural convection MHD past an inclined plate with variable surface heat and mass flux, Int. J. Heat Mass Transfer. 47 (2004) 4449-4457.

[13] M.A. Seddeek, Effect of radiation and variable viscosity on MHD free convection flow past a semi-infinte flat plate with ab aligned magnetic field in the case of unsteady flow, Int. J. Heat Mass Transfer. 45 (2002) 931-935.

[14] P. Dulal, M. Hiranmoy, Influence of thermophoresis and soret-dufour magnetohydrodynamic heat and mass trnasfer over a non-isothermal wedge with thermal radiation and ohmic dissipation, J. of Magnetism and Magnetic Materials. 331 (2007) 250-255.

[15] M.A.A. Mahmoud, Thermal radiation effects on MHD flow of micro-polar fluid over a stretching surface with variable thermal conductivity, Physica A: Statistical Mechanics and its Applications. 375(2) (2007) 401-410

[16] Promise Mebine, Rhoda H. Gumus, On steady MHD thermally raidating and thermosolutal visocus flow through a channel with porous medium, Int. J. Mathematics and Mathematical Sciences. (2010) article ID 287435. doi:10.1155/2010/287435.

[17] E.M Aboeldahab, M.S. El Gendy, Radiation effect on MHD free convective flow of a gas past a semi-infinite vertical plate with variable thermophysical properties for high-temperature differences, Can. J. Phys. 80 (2002) 1609-1619.

[18] A. Md Miraj, Md Abdul A., S. A. Laek, Conjugate effects of radiation and joule heating on Magnetohydrodynaics free convection flow along a sphere with heat generation, American Journal of Computational Mathematics. 1 (2011) 18-25.

[19] A.M. Salema, R. Fathy, Effectsof variable properties on MHD heat and mass trnasfer flow near a stagnation point toward a stretching sheet in a porous medium with thermal radiation, Chin. Phys. B. 21.5 (2012) 0547011.

[20] W.J. Minkowyez, A. Haji-Sheikh, Heat transfer in parallel plate and circular porous passages with axial conduction, Int. J. Heat and Mass transfer. 49 (2006) c2381-2390.

[21] Md. A. Hossain, Md. S. Munir, Mixed convection flow from a verticla flat plate with temperaturedependent viscosity, Int. J. Thermal Sci. 39 (2000) 635-644.

[22] I.A Hassanien, T.H. Al-arabi, Non-darcy unsteady mixed convection flow near the stagnation point on a heated vertical surface embedded in porous medium with thermal radiation and variable viscosity, Comm, Nonlinear Sci. Numer Simulat. 14 (2009) 1366-1376.

[23] B.R. Sharma, Hemanta Konwar, MHD flow, heat and mass transfer about a horizontal cylinder in porous medium. Internation Journal of Innovative Research in Sciences, Engineering and Technology. 3(10) (2014) 16459-16469. DOI: 10.15680/IJIRSET.2014.0310008. 
[24] S.S. Motsa, Z.G. Makukula, On spectral relaxation method approach for steady von karman flow of a reiner-rivlin fluid with joule heating, viscous dissipation and suction/injection. Cent. Eur. J. Phys. 11 (2013) 363-374.

[25] S.S Motsa, A new spectral relaxation method fora similarity variable nonlinear boundary layer flow systems, Chemicla Engineering Communications. 201 (2014) 241-256.

[26] S.S. Motsa, P.G. Dlamini, M. Khumalo, Solving hyperchaotic systems using the spectral relaxation method, Abstract and Applied Mathematics. 12 (2012) Article ID 203461.

[27] S.S. Motsa, P.G. Dlamini, M. Khumalo, A new multistage spectral relaxation method for solving chaotic initial value systems, Nonlinear Dyn. 72 (2013) 265-283.

[28] K.K.P. Sivagnana, R. Kandasamy, R. Savaranan, Lie group analysis for the effect of viscosity and thermophoresis particle deposition on free convective heat and mass transfer in the presence of suction/injection, Theoritical and Applied Mechanics. 36 (2009) 275-298.

[29] S.S. Motsa, P.G. Dlamini, M. Khumalo, Spectral relaxation method and spectral quasilinearization method for solving unsteady boundary layer flow problems, Advances in Mathematicla Physics. (2014) Article ID 341964.

[30] S.S. Motsa, V.M. Magagula, P. Sibanda, A bivariate chebyshev spectral collocation quasilinaerization method for nonlinear evolution parabolic equations, The Scientific World Journal. (2014) Article ID 581987.

[31] C. Canuto et al., Spectral methods in fluid dynamics, Springer-Verlag, Berlin, 1988.

[32] L.N. Trefethen, Spectral Methods in MATLAB, SIAM 2000. 